Estudios sobre armas antiguas, arte militar y vida cultural en oriente y occidente XXX (2010), pp.61-78 ISSN : 0436-029X

doi: $10.3989 /$ gladius.2010.0003

\title{
LOS HALLAZGOS DE ARMAS EN LOS CAM PAMENTOS ROMANOS ALREDEDOR DE NUMANCIA
}

\author{
WEAPON FINDS IN ROMAN CAMPSAROUND \\ NUMANTIA (SORIA, SPAIN)
}

POR

MARTIN LUIK*

\section{Resumen - A bstract - Résumé}

Este artículo pretende ofrecer un panorama general de los hallazgos de armas en los campamentos romanos al rededor de N umancia (línea de circunvalación en torno al oppidum, R enieblas). L os descubrimientos proceden casi en su totalidad de las excavaciones que se realizaron en los años 1906 a 1912 bajo la dirección de A dolf Schulten. Desde entonces, sólo hay que sumar algunos hallazgos aislados. La totalidad de los objetos ha sido documentada y sometida a un nuevo estudio científico y, finalmente, se presentó en forma de monografía en el año 2002 (L uik, 2002 con resumen español: 173-sq). En general, Ilama la atención la reducida proporción de armas defensivas (corazas de discos, cotas de malla, umbo de escudos). Por el contrario, las armas ofensivas y arrojadizas están ampliamente representadas: puntas de lanzas, pila de lengüeta y de enmangue tubular y los más variados tipos de puntas de proyectiles. M enos representadas se hallan, en cambio, las espadas y los puñales, junto con sus accesorios correspondientes. En suma, los presentes hallazgos ofrecen buena muestra de los pertrechos y de las armas del ejército romano de la República tardía. A I mismo tiempo, los hallazgos brindan también información sobre la estructura interna del ejército romano de esta época.

This paper provides an overview of the finds of arms and armour from the Roman camps around N umantia, i.e. the circumvallation surrounding the oppidum of Renieblas. Virtually all of the material presented was recovered during the 1906-1912 excavations led by A dolf Schulten. Since the conclusion of these research campaigns, there have only been isolated surface finds. The entire corpus of finds was drawn and - in part - photographed. The material was furher analysed and published as a monograph (Luik 2002). A particularly interesting result of this analysis is that there are relatively few defensive items such as body-armour or shield bosses. Offensive weaponry, and ranged weapons in particular, on the other hand, are frequent and include spearheads, socketed as well as tanged pila and various types of arrow and bolt heads. Interestingly, there are few swords or daggers, and little associated equipment parts. A II in all, the presented finds provide a unique insight into the equipment and arms of the R oman A rmy during the Late R epublic. At the same time, the corpus of material discussed and presented provides new information regarding the internal structure of the Roman A rmy at the time.

Cet article entend présenter un panorama general des trouvailles d' armes sur le site des camps romains autour de Numance, tant sur la circonvallation entourant I'oppidum lui-même qu'à R enieblas. Dans leur quasi-totalité, les découvertes remontent aux fouilles menées entre 1906 et 1912 sous la direction d'A dolf Schulten. Depuis Iors, on ne compte que quelques trouvailles isolées. La totalité des objets a été cataloguée et a fait l'objet d'un réexamen scientifique, publié sous forme de monographie en 2002 (L uik, 2002, avec résumé en espagnol: 173 sq.). D'une façon générale, on y note la faible proportion d'armes défensives (disques-cuirasses, cotes de mailles, umbos de

* L udwig-M aximilians-U niversität M ünchen. Institut für Vor- und Frühgeschichtliche A rchäologie und Provinzialrömische A rchäologie. Geschwister-Scholl-Platz 1. D-80539 M ünchen. e-mail: M artin.Luik@ vfpa.fak12.uni-muenchen. de. 
boucliers). A u contraire, les armes offensives, et notamment les armes de jet, sont abondamment représentées: pointes de lances, pila à soie ou à douille, ainsi que toutes sortes de pointes de projectiles. En revanche, les épées et les poignards, ainsi que leurs accessoires, sont peu nombreux. A insi, le matériel numantin offre un échantillon assez représentatif des armes de l'armée romaine de la République tardive. Dans le même temps, ces trouvailles nous apportent aussi des informations sur la structuration interne de l'armée romaine de cette époque.

\section{Palabras Clave - Keywords - Mots clés}

Numancia; Renieblas; campamentos; armas ofensivas; armas defensivas; ejército romano; época tardorrepublicana.

Numantia; Renieblas; Roman camps; A rms and armour; Roman army; Late Roman Republic.

Numance; Renieblas; camps; armes offensives; armes défensives; armée romaine; fin de la République.

L os hallazgos que se tratarán a continuación se descubrieron durante las excavaciones que se realizaron en los años 1906 a 1912 en los campamentos romanos en torno a Numancia y en los campamentos de Renieblas, ocho km al este de los primeros (Schulten, 1927; id., 1929; Cadiou, 2008: 296-sq.; Dobson, 2008: 14-sq.; L uik, 2009: 53-sq.). Todo el material hallado se conserva actualmente en el Römisch-Germanisches Zentralmuseum de M aguncia (RGZM), donde se volvió a estudiar científicamente en los años 1994 a 1997, en el marco de una beca de investigación otorgada por el Deutsches A rchäologisches Institut (Instituto A rqueológico Alemán) y el RGZM (Luik, 2002). En este trabajo intervinieron especialistas de diversos ámbitos que dibujaron nuevamente la totalidad de los hallazgos existentes. También se fotografiaron las piezas más importantes. Sólo en algunos casos excepcional es de deterioro o pérdida absoluta de los vestigios arqueológicos fue necesario reproducir los dibujos antiguos. A las excavaciones realizadas hace cerca de cien años hay que añadir al gunos hallazgos aislados, de los cuales la mayoría fueron publicados por F. M orales Hernández en el año 1995 (M orales Hernández, 1995: 127 -sq. y 242 -sq.).

Por aquel entonces, los trabajos sobre el terreno estaban bajo la dirección de A dolf Schulten (1870-1960), catedrático de Historia A ntigua en la U niversidad de Erlangen (Schulten, 1931, 29-sq.; 1953: 35-sq.; Blech, 2007; Dobson, 2008: 122-sq. et 235-sq.). Schulten estaba secundado por todo un equipo de reconocidos especialistas, como Constantin K oenen, quien excavara durante muchos años en el campamento de legionarios de Novaesium/N euss, Walter Barthel, poco después director de la Comisión romano-germánica (R ömisch-Germanische Kommission) de Frankfurt/M ain, pero desgraciadamente desapareciendo en 1915 en el frente del O este (von Schnurbein, 2002: 145-146) y Ernst Fabricius, catedrático de Historia A ntigua en Friburgo de Brisgovia.

M ediante las excavaciones en el yacimiento, Schulten se proponía comprobar la credibilidad de las fuentes escritas, en primer lugar las $\mathrm{H}$ istorias de Polibio, pero también la I beriké de A piano (L andfester, 2007; K uhn-Chen, 2007). A simismo, se esperaba obtener abundante documentación sobre la historia del ejército romano de la República y la cultura material de los celtíberos (Schulten, 1931: 12-13).

El resultado de mayor trascendencia de las excavaciones de 1906 a 1908 fue el descubrimiento de una línea de circunvalación al rededor del oppidum de N umancia, protegido en total por siete campamentos y dos fortalezas a orillas del río (Luik, 1997: 223-sq. no 6; M orales Hernández, 2007). El hallazgo de estas construcciones defensivas Ilevó a Schulten a vincularlas históricamente con el cerco y la conquista de Numancia por parte de P. Cornelio Escipión Emiliano en los años 134/133 a.C. En el cerro de Castillejo se identificaron tres periodos de construcción, de los cuales el más reciente se interpretó como el cuartel general de Escipión Emiliano (Schulten, 1927: 167-sq.). L os dos períodos de construcción más antiguos se asociaron a las campañas contra N umancia de M . Claudio M arcelo en 152/151 a.C. y de Q. Pompeyo 
A ulo en 141/140 a.C. ( L uik, 2007a: 60-sq., fig. 78a et 73). D esde entonces, las investigaciones de A Ifredo J imeno, Fernando M orales y M ike D obson han conducido, con especial intensidad en los últimos años, a algunas modificaciones que no abordaremos aquí con más detalle por razones de espacio (J imeno, 2002: 169-sq.; 2006: 176-sq.; M orales Hernández, 2007: 266-sq.; Dobson, 2008: 387-sq., fig. 269).

Entre 1909 y 1912, las excavaciones se extendieron hasta los campamentos romanos próximos a Renieblas, donde se pudo constatar una sucesión de al menos cinco establecimientos sucesivos (L uik, 1997: 227-sq., no 7; L uik, 2007b). Schulten propuso las siguientes dataciones para dichas construcciones, basándose únicamente en consideraciones históricas: campamento I, 195 a.C., campamento II, desconocido, campamento III 153 a.C., campamento IV 75 a.C., campamento V 75/74 a.C. (Schulten, 1929: 33-sq.; 41-sq.; 137-sq.; 180-sq.). L os hallazgos menores descubiertos en el curso de las excavaciones siguientes no influyeron en sus primeras consideraciones. Otras investigaciones más recientes han completado los datos antiguos sobre tales yacimientos. Por encargo del Instituto A rqueológico A lemán, en los años 1997 a 2001 se volvieron a medir las estructuras del campamento $V$, en al gunos casos extraordinariamente bien conservadas (L uik y M üller, 2006). Por su parte, M ike Dobson ha realizado recientemente una nueva numeración de cada uno de los periodos de los campamentos (campamento I-V II) (2008: 125-sq.). U no de los aspectos que ha generado mayor controversia entre los investigadores es la cronología que Schulten atribuye a los campamentos IV y V: recientemente se ha sugerido en más de una ocasión que éstos pudieran datar de finales del siglo II a.C. o incluso de principios del siglo I a.C. (Dobson, 2008: 35-sq.; Dobson y M orales, 2008: 224).

En el marco de este artículo sólo podemos incluir algunas observaciones breves acerca de la metodología de las excavaciones antiguas. Para más información, remito a la amplia literatura de los últimos años (Blech, 1995: 38-sq; 2007: 31-sq.; Luik, 2002: 7-sq.; D obson, 2008: 14-sq.; 28-sq.). Por aquella época, hace cien años, estas investigaciones podían considerarse ejemplares, aunque hoy día no satisfagan las exigencias actuales en diversos campos. Como se puede deducir de planos y fotografías antiguos, las excavaciones se llevaron a cabo principalmente con ayuda de estrechas zanjas de prueba, omitiendo en gran parte la exploración de superficies contiguas mayores. Probablemente, esta forma de proceder respondía al estado de la técnica de excavación del momento, pero también al deseo de alcanzar pronto resultados y obtener la mayor cantidad de hallazgos posible. No obstante, es de temer que, de este modo, hayan quedado sin documentar muchos hallazgos. L os diarios de las excavaciones que se han conservado contienen sobre todo breves indicaciones sobre las circunstancias externas (clima, visitantes, etc.). Tanto los dibujos esquemáticos del emplazamiento de los hallazgos como las observaciones sobre los descubrimientos concretos, para los cuales en muchos casos ya no parece viable determinar una localización indiscutible, son asimismo escasos. Por esta razón, en las publicaciones apenas hay datos sobre la ubicación concreta del lugar del hallazgo de las diferentes piezas y sobre posibles interrelaciones, como complejos cerrados o hallazgos accesorios importantes, tales como monedas o cerámica. Con frecuencia, el subsuelo es tan rocoso-pedregoso que no puede darse una sucesión clara de estratos, como sucede en la parte norte de R enieblas. Por ello, deben ponerse en duda en muchos casos las adscripciones de los hallazgos a determinados periodos de los campamentos que hace Schulten.

L a publicación de tales descubrimientos se confió a acreditados expertos de la época. De la presentación de los objetos de metal se ocupó M aximilian von Groller-M ildensee (1838-1920), quien excavara durante muchos años en Carnuntum y en Lauriacum, A ustria. Sin embargo, su trabajo no presentaba una clasificación por grupos de objetos, como es habitual hoy en día, sino en función de las diferentes campañas de excavación. Siguiendo este procedimiento, en el tercer y cuarto volumen de la serie Numancia se dio a conocer la mayoría de las piezas de 
metal (Schulten, 1927: 243-sq.; 1929: 201-sq.). Sin embargo, con este método tan particular, resulta sumamente difícil obtener una visión de conjunto de la composición del material haIlado. Por otra parte, K oenen presentó muy pocas piezas de los objetos de cerámica (Schulten, 1929: 248-sq.). A ello se añade que, en aquellos tiempos, apenas se conocían yacimientos apropiados para realizar estudios comparativos, Io que habría facilitado en gran medida el estudio de un material tan importante. AI parecer, todos los textos se terminaron, como muy tarde, hacia finales de la Primera Guerra M undial, en 1918. D ebido a las consecuencias de la guerra, la publicación se retrasó más de una década (Schulten, 1927; 1929).

Como ya se ha mencionado, la totalidad de los hallazgos se volvió a estudiar científicamente en los años 1994 a 1997 y, finalmente, en 2002, el autor de este artículo los presentó en el contexto de una monografía. Para ello también se tuvieron en cuenta los descubrimientos aislados de décadas anteriores. Dicho volumen contiene asimismo todas las referencias necesarias a hallazgos comparables y una abundante bibliografía especializada. Por motivos de espacio, en el presente artículo solo mencionaremos los paralelismos más relevantes y ofreceremos una selección de la bibliografía especializada publicada desde entonces. En este trabajo se pretende ofrecer un panorama general de los hallazgos de armas en los campamentos romanos al rededor de Numancia, con especial atención a los aspectos siguientes:

Se presentarán en particular los distintos tipos de armas que están representados en los campamentos romanos en torno a N umancia, indicándose asimismo de qué regiones provienen. L as posibilidades son, fundamentalmente, Italia, Ia Península Ibérica, Europa Central, Á frica y O riente Próximo. Por otra parte, se analizará a qué fase de la evolución de la historia de los diferentes tipos de armas pertenecen los hallazgos de los campamentos romanos numantinos. ¿Qué conclusiones pueden extraerse de todo ello acerca de la estructura interna del ejército romano? (Cagniart, 2007).

\section{LAS ARMAS DEFENSIVAS}

En los campamentos romanos al rededor de N umancia no se han encontrado muchas armas defensivas. Los discos de coraza merecen especial atención (L uik, 2002: 69-sq.), sobre todo por el hallazgo de varias láminas de bronce. Entre ellas, hay que señalar en primer lugar el hallazgo de un disco en Castillejo, cuyo anverso está decorado con un motivo ornamental a base de ranuras concéntricas (fig. 1,1). A lo largo del borde exterior aparecen varios orificios, separados entre sí por dos $\mathrm{cm}$, mediante los que la pieza se sujetaba presumiblemente a una base de cuero o tela. En la parte superior, los orificios estaban cubiertos por cabecitas de bronce semiesféricas. En la lámina aparecía con claridad una rotura. En una parte, se había colocado una lámina rectangular ricamente decorada. En el dibujo aparece una lámina montada que es un añadido posterior. EI mejor paralelo lo constituye el hallazgo de un disco en la tumba 1 de Troccola, Pietrabbondante (I talia), que, a causa de los demás hallazgos de la fosa, fue datado, sin embargo, en una época mucho más temprana, a saber, ya en la primera mitad del siglo V a.C. (Tomedi, 2000: 94 № 399. 400 tabla 144).

A demás de esta lámina, en Renieblas se encontraron otras tres de bronce, sin adornos y planas, que miden $12 \mathrm{~cm}$ de diámetro. En cuanto a las dimensiones, estas placas de bronce sin decoración no se asemejan a los discos de coraza conocidos de la Península I bérica, cuyo tamaño es muy variable, oscilando entre 15 y $25 \mathrm{~cm}$. Sin embargo, en sus Historias (6.23.14), Polibio narra inequívocamente que los soldados romanos llevaban esta clase de discos, en concreto, los pertenecientes a las capas sociales más bajas. No obstante, hasta el momento apenas se han hallado piezas análogas en campamentos romanos, con excepción de los de Numancia. 


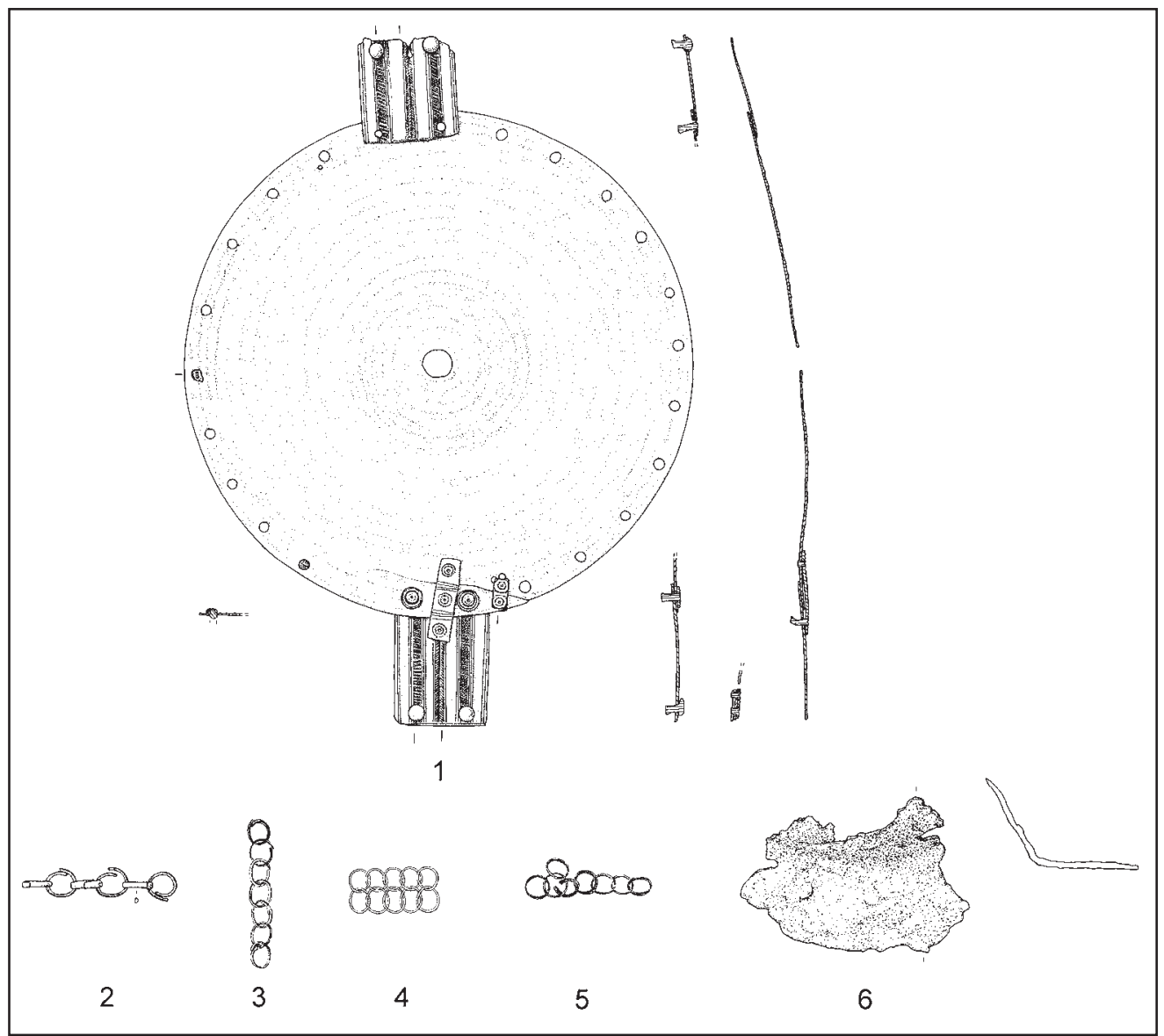

Figura 1. Los hallazgos de armas en los campamentos romanos alrededor de Numancia. 1 corazas de disco, 2-5 fragmentos de cotas de malla, 6 umbo de escudo. Escala 1:3.

Esta observación no debe sorprender a la vista de la deficiente situación de la investigación (Bishop y Coulston, 2006: 63-sq.). De modo general, se debe tener en cuenta que cuando Polibio escribió su obra, los datos de que disponía se habían quedado ya obsoletos (Walbank, 1979: 775).

En los campamentos romanos al rededor de Numancia se han encontrado en repetidas ocasiones pequeños aros de bronce, formados por finas bandas de chapa acanalada de forma redondeada-oval ada, ensartados unos en otros (fig. 1, 2-5). El diámetro es de 0,9-1,1 cm (L uik, 2002: 73-74). Es muy probable que estos aros provengan de cotas de malla (Viand, 2008: 36sq., fig. 12, tipo A/C; Poux, 2008: 348-sq.). Polibio cuenta que en el siglo II a.C. Ios miembros acaudalados del ejército romano llevaban cotas de malla (Pol. 6.23.15). Tanto en el monumento a la victoria de Publio Emilio en Delfos, Grecia (167 a.C.), como en el relieve de los guerreros de Estepa, en la provincia de Sevilla, datado en la primera mitad del siglo I a.C. (B lech, 1993: 268-269, tabla 31), pueden verse las correspondientes representaciones escultóricas.

En Renieblas se halló el fragmento de un umbo de hierro (Luik, 2002: 75) (fig. 1, 6). Al parecer, perteneció originariamente a un escudo ovalado con una nervadura en forma de huso. Como prueban las representaciones de la llamada A ra de Domicio A henobarbo o el mencionado relieve de los guerreros de Estepa, Ios soldados romanos llevaban este tipo de escudo (Bishop y Coulston, 2006: 61-62; Poux, 2008: 344-sq., fig. 31). 


\section{ARMAS OFENSIVAS}

Tal como cabría esperar de la misión de cercar y sitiar una ciudad fortificada, en los campamentos romanos de los alrededores de Numancia se ha encontrado un amplio espectro de armas ofensivas y arrojadizas.

Entre las puntas de lanza, se pueden diferenciar al menos cuatro tipos distintos (L uik, 2002: 76) (fig. 2). Todos tienen en común el asta de tubo y la hoja visiblemente rebajada. Entre el material hallado, dominan claramente las lanzas que presentan una hoja con un corte transversal muy plano o con un nervio central no muy marcado (fig. 2, 1). Estas lanzas son frecuentes en los yacimientos de la R epública tardía y de comienzos del Imperio. L lama la atención la presencia de dos puntas de proyectil con hoja corta y triangular (fig. 2, 2). A I menos en estos dos casos es más probable que se trate de jabal inas. De todos modos, es prácticamente imposible establecer una diferenciación inequívoca entre lanzas y jabalinas. En cuanto al número de piezas, pocas son las típicas puntas de lanza del entorno de La Tène, con el nervio central muy

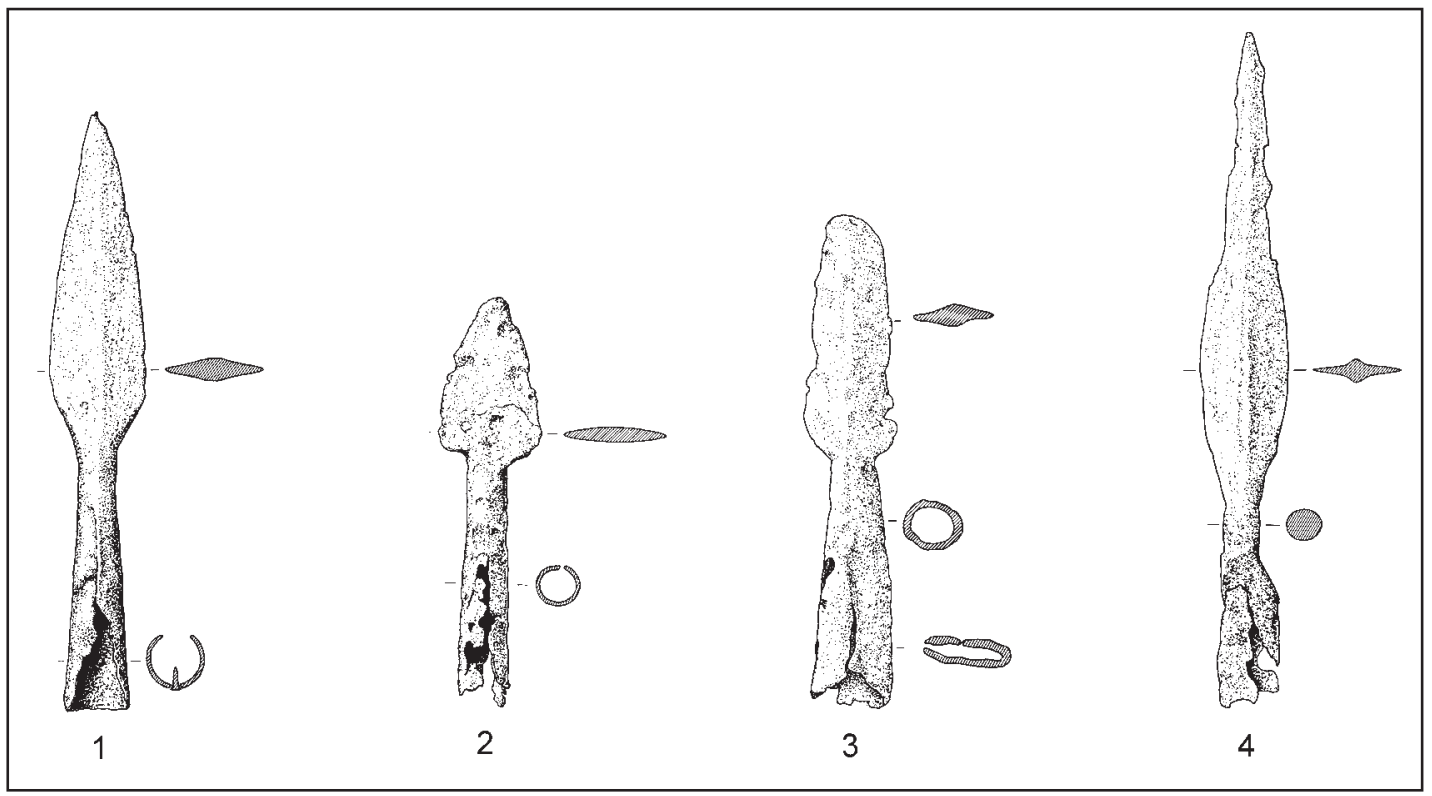

Figura 2. Los hallazgos de armas en los campamentos romanos alrededor de Numancia. Puntas de lanzas y jabalinas. Escala 1:3.

marcado (fig. 2, 3). Tan sólo una lanza de Renieblas tiene los lados de la hoja curvados y por este motivo podría ser de origen celtíbero (fig. 2, 4). En general, se aprecian muchas características comunes con los hallazgos de Cáceres el V iejo. En cambio, si las comparamos con las lanzas de las fortificaciones de A lesia, donde predominan las puntas de lanza de hoja estrecha y con un marcado nervio central, las diferencias son notables.

A parte de las puntas de lanza, son sobre todo los numerosos hallazgos de pila los que suscitan especial atención (L uik, 2002: 76-sq.) (fig. 3). Como mínimo nueve ejemplares encontrados en los campamentos romanos al rededor de Numancia pertenecen al tipo de pilum de lengüeta (fig. 3, 1-2). Dos ejemplares, de $74 \mathrm{~cm}$ y $54 \mathrm{~cm}$ de largo respectivamente, se han 
conservado incluso completos. Como es obvio, las piezas son relativamente grandes. En los casos en los que se ha conservado la lengüeta de sujeción, ésta es plana, de $9 \mathrm{~cm}$ de largo y 5,5 $\mathrm{cm}$ de ancho, y está provista de dos orificios, practicados a una distancia de $3,5 \mathrm{~cm}$. Tan solo en un caso se puede comprobar con seguridad que la lengüeta estaba doblada hacia un lado. $L$ a hoja del pilum de lengüeta tiene en general forma redondeada. L a relación entre el tamaño de la lengüeta de sujeción y la hoja es 1:4 o más; aunque las puntas de los pila pueden ser de muchos tipos, predominan claramente las formas alargadas.

M ención especial merecen dos pila encontrados en Renieblas, por su construcción particular (fig. 3, 3-4). En uno de ellos, un extremo de la hoja termina en un ensanchamiento en forma de botón. Este hallazgo puede compararse con un ejemplar completo del campamento de Cáceres el Viejo en el que el botón parece indicar que formaba parte del extremo inferior de la varilla (U Ibert, 1984: 107, tabla 24, 187). Esta estructura era necesaria para unir de forma segura la hoja de hierro al asta de madera (Connolly, 1997: 49). Los dos ejemplares constituirían, por tanto, pruebas tempranas de la sujeción de las hojas de hierro con abrazaderas metálicas, que más tardíamente se encuentran con mucha frecuencia (Feugère, 1993: 166-sq.; Connolly, 2001-2002: 1-2; Bishop y Coulston 2006: 73-sq.; Poux, 2008: 332-sq.). Entre los pila de enmangue tubular de los campamentos romanos al rededor de N umancia destacan al gunos ejemplares más bien grandes (fig. 3, 5). En casos extremos, la longitud puede llegar hasta $95,5 \mathrm{~cm}$. La hoja de este tipo de pilum, que sobresale progresivamente de la empuñadura, es redonda o cuadrangular y la punta alargada y afil ada. L a mayor parte de las restantes puntas de proyectil, que probablemente pertenezcan a los pila de lengüeta o de enmangue tubular, son de forma piramidal. M uchas presentan leves daños causados por el impacto y están ligeramente quebradas en la parte delantera. Se han hallado muy pocos ganchos.

A simismo existe una variante más pequeña en la que el asta tubular se transforma en una hoja larga, de aspecto macizo y forma cuadrangular, en la que fal ta una de las puntas rebajadas (fig. 4, 1). Esta variante mide $20-30 \mathrm{~cm}$ de largo, aproximadamente, y pesa entre 80 y $130 \mathrm{gr}$. En el pilum más corto, M. von Groller-M ildensee creyó identificar la jabalina de los velites (Schulten, 1929: 204).

A modo de resumen cabe decir que en los campamentos romanos al rededor de N umancia no se hallan representadas formas de pila más antiguas que las del tipo de Telamón. La lengüeta de sujeción puede ser oblongo-rectangular y doblada hacia un lado (variante 1) o bien ovalada (variante 2). Estos pila presentan una forma relativamente compacta y son más pequeños, de hasta $40 \mathrm{~cm}$. La punta es, en general, plana, triangular, y muchas veces está provista de un garfio. La referencia más segura para su datación la proporciona el hecho de haber sido hallados en el oppidum de Castell ruf, que fue abandonado hacia el año 200 a.C. (Á Ivarez A rza y Cubero A rgente, 1998). A simismo es probable que los hallazgos procedentes del yacimiento epónimo de Telamón pertenezcan también a una época similar (L uik, 2000: 270-sq., fig. 1-2). Seguramente, estos pila guardan relación con la batalla contra los galos que tuvo lugar allí en el año 225 a.C. (Luik, 2000: 269). Por otro lado, el hallazgo de Entremont prueba que, hacia finales del siglo II a.C. (alrededor de 120/100 a.C.), estaban aún en uso los pila del tipo de Telamón.

Hallazgos análogos en los campamentos romanos alrededor de Numancia demuestran sin lugar a dudas que tanto los pila de enmangue tubular como los de lengüeta (el tipo más reciente) se utilizaban a la vez en esa época en el ejército romano (Connolly, 2001-2002: 1-sq.; Bishop y Coulston, 2006: 50-sq.; Quesada Sanz, 2007: 379-sq.). En el caso del pilum de enmangue tubular pueden constatarse con seguridad varios tipos. Para realizar tentativas de subclasificaciones más razonadas faltan aún más hallazgos (referencia: la reforma del ejército de $\mathrm{M}$ ario). El ensamblaje seguro entre la parte superior de hierro y la inferior de madera 


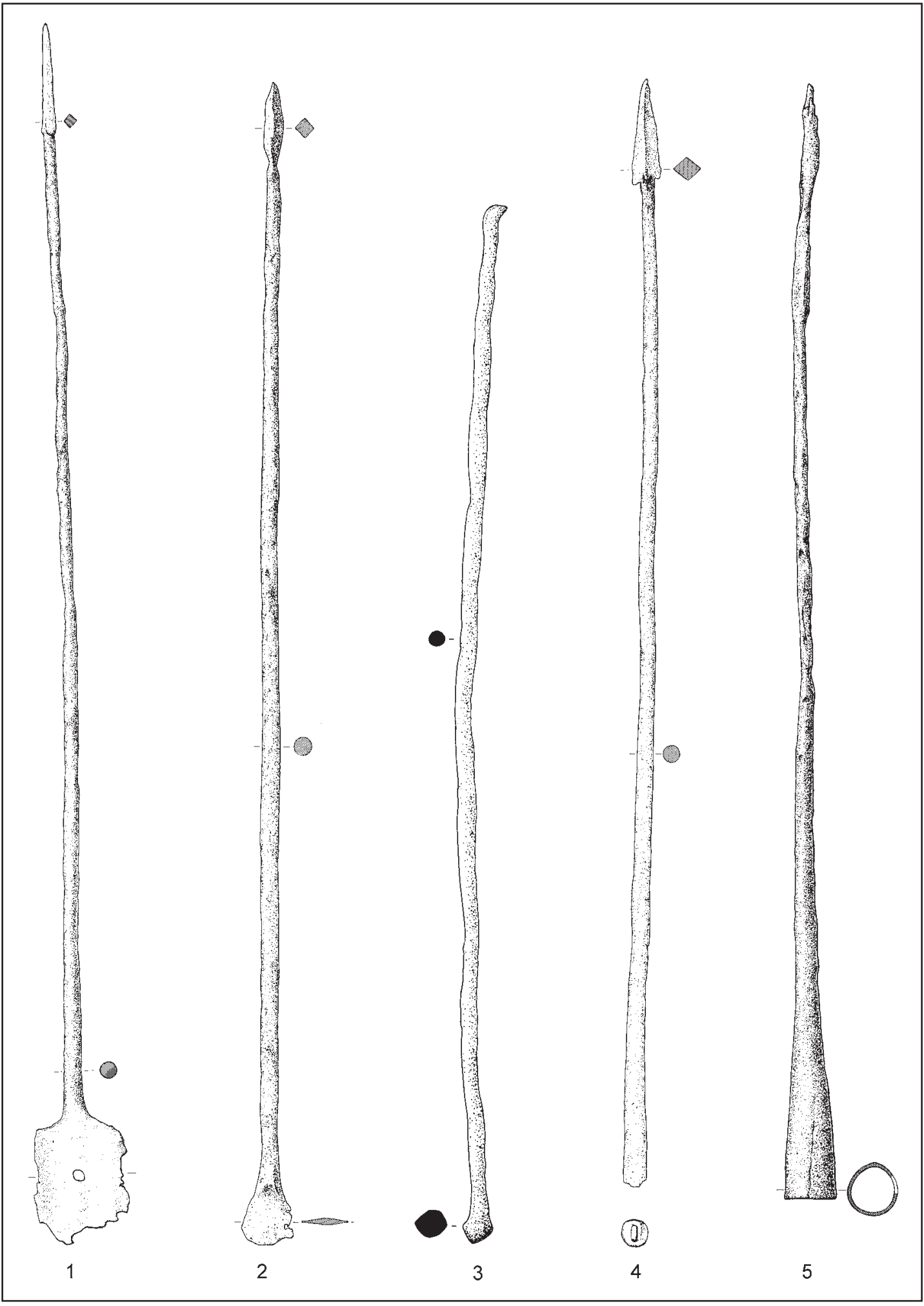

Figura 3. Los hallazgos de armas en los campamentos romanos al rededor de N umancia. Pila. Escala $1: 3$.

Gladius, XXX (2010), pp. 61-78. ISSN : 0436-029X . doi: 10.3989/gladius.2010.0003 
fue siempre problemático y requería permanentes modificaciones e intentos de mejora. En la actualidad, la mayoría de los investigadores piensa que el pilum de lengüeta representa la forma más reciente de este tipo de arma y que muy probablemente fue traído de I talia por los romanos (Luik, 2000: 275; 2002: 91; Bishop y Coulston, 2006: 52; Quesada Sanz, 2007: 386387). En cuanto al origen del pilum de enmangue tubular, técnicamente menos sofisticado, se consideran en igual medida varias regiones, entre ellas, la Península Ibérica. Es posible que este enigma no llegue a resolverse nunca con certeza absoluta.

Otra arma arrojadiza con asta tubular se distingue por tener una hoja larga y cuadrangular, que se ensancha claramente a partir del asta (L uik, 2002: 81) (fig. 4, 2). Estas puntas de proyectil son más delgadas y ligeras, miden hasta $20 \mathrm{~cm}$ de largo y pesan entre 40 y $55 \mathrm{gr}$. Quizá pertenecieran a armas de soldados de caballería, pero atendiendo a su forma un tanto peculiar y a su reducido peso, podrían constituir también puntas de catapulta (cf. Poux, 2008: 358-sq.). En las puntas de catapulta se distinguen cuatro categorías de peso (fig. 4, 3-6). En el material hallado se encuentran representadas por piezas individuales (L uik, 2002: 82-sq.; panorámica general: Sáez A bad, 2005: 155-sq.; B ishop y Coulston, 2006: 58-sq.; García Díez y Sáez A bad, 2007: 455-sq.; Poux, 2008: 353-sq.). Un primer grupo destaca por una cabeza compacta cuadrangular. El peso es bastante homogéneo, entre 43 y $50 \mathrm{gr}$. En otros dos grupos, las cabezas de proyectil son algo más pequeñas; el peso es, lógicamente, menor. Por último, en un cuarto grupo resulta especialmente llamativa la cabeza pequeña, romboidal, y el peso de 21 gr. Estas cuatro categorías coinciden sorprendentemente con las puntas de proyectil del hallazgo de armas de Ephyra, Epiro (B aatz, 1982: 230-231, fig. 7). De hecho, los dos tipos de proyectiles mencionados en primer lugar representan las formas típicas de la República tardía.

L as puntas de flecha con gancho constituyen un grupo especialmente nutrido (L uik, 2002: 84) (fig. 4, 8-11). El tipo más frecuente en los campamentos romanos al rededor de Numancia posee una punta alargada, en la mayoría de los casos provista de un gancho, que se torna llamativamente gruesa en la parte delantera y trasera, en la punta donde se inserta el largo aguijon. M uchas puntas de lanza presentan la punta enrollada debido al impacto. Puntas de flechas similares de La Loma han sido publicadas recientemente (Peralta Labrador, 2007: 497-sq., tabla 2; cf. Poux, 2008: 360-sq.).

En este contexto, no puede omitirse la referencia a las puntas de flecha de tres al etas ( $L$ uik, 2002: 84) (fig. 4, 12-13): los cuatro ejemplares se encontraron en la línea de circunvalación y su peso es de $4 \mathrm{gr}$. aproximadamente. Las puntas de flecha de tres al etas podrían estar vinculadas con la presencia de arqueros libios, como atestigua A piano con ocasión del cerco del oppidum en el año 134/133 a.C. (A p. Ib., 89.387). Entre las restantes puntas de proyectil, mención especial merecen las sencillas flechas de hierro alargadas (fig. 4, 14-15) (D eyber, 2008), pero también formas más compactas cuadrangular-piramidales, fabricadas asimismo en hierro (fig. 4, 16).

Finalmente, algunas observaciones acerca de los proyectiles para hondas y los proyectiles de piedra (Luik, 2002: 85-86): Ios cuatro proyectiles de plomo para hondas hallados son de forma bicónica (fig. 4, 17). Dos de ellos presentan un contorno más bien anguloso, los otros dos, redondeado. U na vez más, dos proyectiles de plomo tienen, con 32,7 gr, el mismo peso. El tercero, de $27 \mathrm{gr}$, es algo más liviano. Con ello, los cuatro proyectiles de plomo se cuentan entre los ejemplares de la A ntigüedad más ligeros que se conocen. En general, la presencia de honderos en el ejército romano está documentada incontestablemente (cf. también Gruat et alii, 2003, panorámica general: K avanagh de Prado y Quesada Sanz, 2007: 75-sq.; Poux, 2008: 365-sq.). Junto a ellos, en los campamentos de la línea de circunvalación se han encontrado también cuatro proyectiles para hondas hechos de barro (fig. 4, 18). También estos resultan extremadamente livianos. 


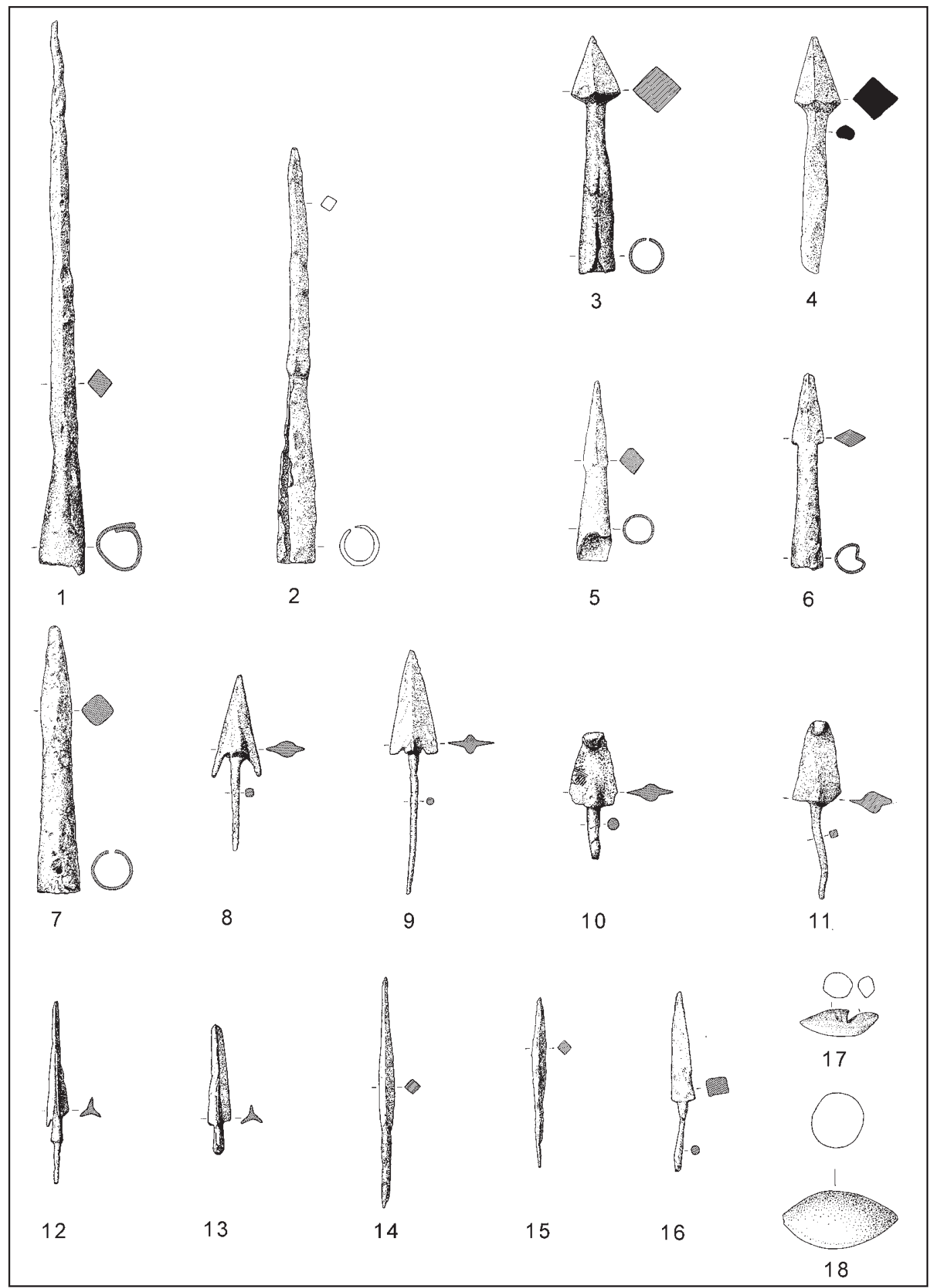

Figura 4. Los hallazgos de armas en los campamentos romanos al rededor de Numancia. Puntas de proyectiles. Escala 1:3. 
Todos los proyectiles de piedra están fabricados con arenisca originaria de la zona. En total, Schulten diferenció cuatro calibres: la más pesada (calibre 1) de 10 minas ( $n$ - 1-4), calibre 2 de 3 minas ( $n-5$ ), calibre 3 de 2 minas ( $n-6-9$ ) y calibre 4 de 1 mina ( $n-10$ ). Por su reducido tamaño, es probable que se trate en todos los casos de proyectiles que se lanzaban con las manos. Los ejemplares mayores podrían interpretarse también como munición para catapultas. Esta interpretación tiene en cuenta asimismo el caso de los proyectiles para hondas del hallazgo de Calahorra, que parece pertenecer al contexto histórico del levantamiento de Sertorio (Cinca et alii, 2003; Velaza et alii, 2003).

La incorporación de las catapultas al armamento de las legiones está documentada en las fuentes escritas a partir de mediados del siglo III a.C. (Baatz, 1994: 128). A piano menciona la presencia tanto de arqueros como de lanzadores de piedras durante el sitio de 134/133 a.C.: habrían estado apostados en torres a lo largo de la línea del cerco (A p. Ib., 92.401; cf. Schulten, 1927: 44; 85). En las excavaciones de los campamentos romanos de Castillejo, Peña R edonda, Valdevorrón y de los campamentos III y V de R enieblas (según Schulten) se descubrieron estructuras que Schulten interpretó como ruinas de puestos de tiro (Schulten, 1927: 86, 102-103, 210-sq., 218-219; 1929: 52-sq., 149-sq.).

En cuanto a las espadas largas, se han conservado muy pocas partes de hojas con valor informativo (Luik, 2002: 86-sq.; hoy anticuado, M iks, 2007: 38-sq.). Solamente una hoja de espada hallada en R enieblas se ha conservado mejor (fig. 5, 1). La espiga de la empuñadura es delgada y redondeada. La hoja presenta lomos que caen en diagonal y un nervio central poco marcado. Los dos filos de la hoja discurren casi en paralelo. El ancho disminuye hacia la punta casi imperceptiblemente. Debido a sus características, esta espada debe datarse en la fase media de $L$ a Tène. A este periodo pertenece también el fragmento bien conservado de una vaina de chapa de hierro encontrado también en Renieblas (fig. 5, 2). L a boca de la vaina tiene forma de campana.

A quí cabe mencionar también una vaina que presenta los bordes reforzados por una pieza de hierro, de sección en «U», de media caña, en mal estado de conservación, procedente de Renieblas (fig. 5, 3), y una pieza de bronce hallada en los campamentos de la línea de circunvalación de Numancia (fig. 5, 5). El extremo inferior está formado por un disco plano, circular. En relación con estos ejemplares podemos aducir numerosos hallazgos paral elos procedentes de contextos de la edad del hierro. Estas piezas de armamento provienen presumiblemente del gladius romano. Entre ellas se cuenta además la hebilla rectangular de bronce con los lados remetidos (L uik, 2002: 74-sq.; fig. 170, R44) hallada en Renieblas (véase en general: Poux, 2008: 372-sq.).

Entre los puñales, llama la atención la chapa de la empuñadura de uno de tipo frontón, originaria de R enieblas (L uik, 2002: 87-sq.) (fig. 5, 4). Este tipo de puñal se data normalmente en fechas muy anteriores, en concreto, ya en los siglos V /IV a.C. N o es posible decidir con seguridad si su presencia, unida a otros hallazgos de la misma época, remite a una colonización más antigua de Renieblas durante la Edad del Hierro o si da cuenta de que este tipo de puñal fue utilizado durante más tiempo del que normalmente se supone. En cuanto al resto de los hallazgos, se trata aparentemente, sin excepción, de puñal es biglobulares (fig. 5, 6-7). A unque ciertamente presentan similitudes con los de Cáceres el Viejo, en los campamentos romanos de Numancia predominan más bien los puñales de hojas curvadas. Los lomos discurren rectos o muestran un leve declive. L as nervaduras central es de las hojas están configuradas de forma sencilla, sin ranuras adicionales, como en los puñal es de los campamentos romanos de Cáceres el Viejo y las fortificaciones de A lesia. El puñal de Castillejo (fig. 5, 6) presenta en el lomo tres orificios, lo que es asimismo un fenómeno temprano.

En yacimientos de la edad del hierro de la Península I bérica se ha comprobado la existencia de puñales biglobulares, principalmente en el curso superior del Duero y en la M eseta, da- 


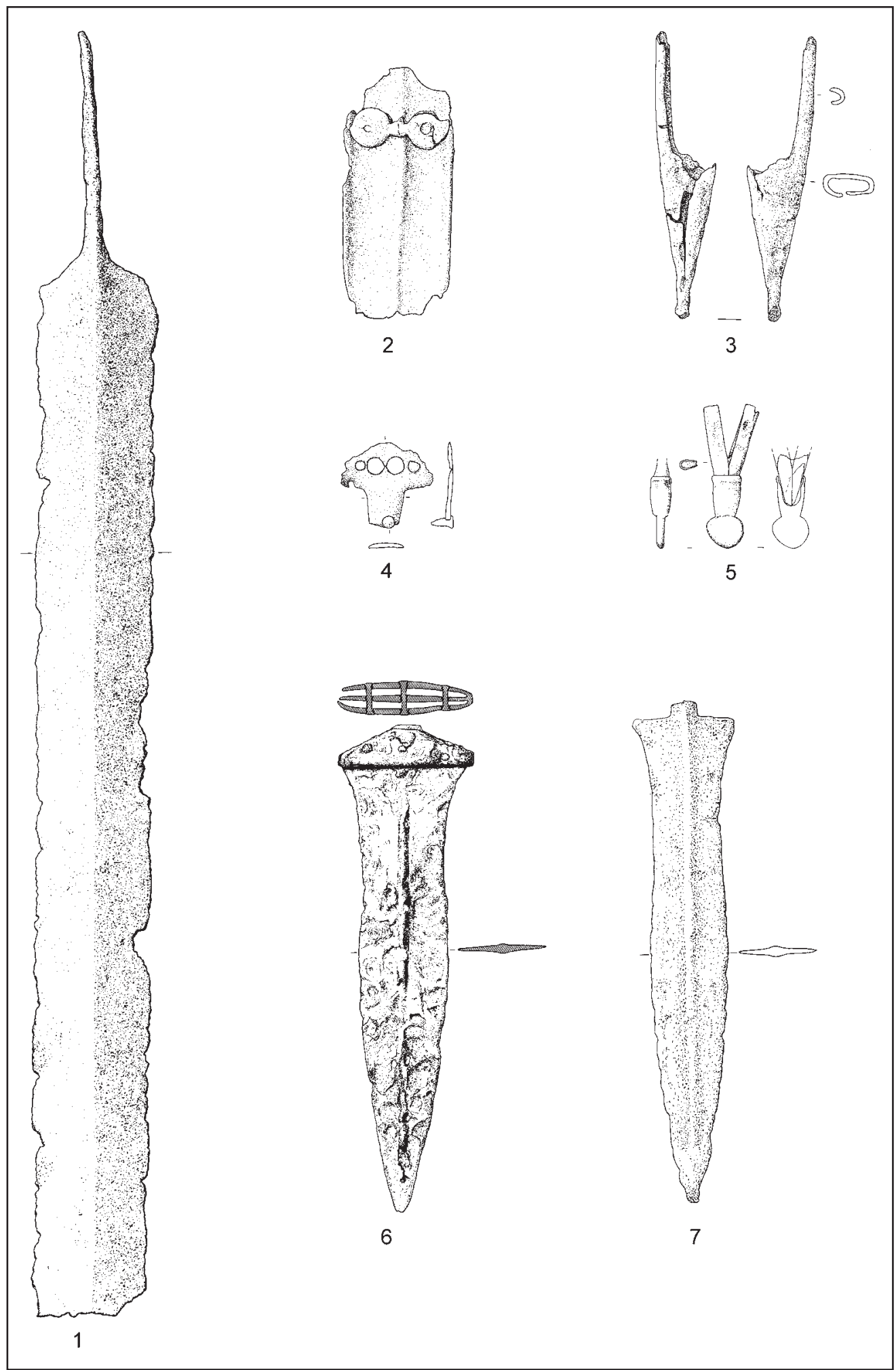

Figura 5. Los hallazgos de armas en los campamentos romanos alrededor de Numancia. Espadas y puñales. Escala 1 1:4, 2-7 Escala 1:3.

Gladius, XXX (2010), pp. 61-78. ISSN : 0436-029X . doi: 10.3989/gladius.2010.0003 
tados a partir mediados del siglo III a.C. A continuación, se utilizó el modelo del pugio romano (Quesada Sanz, 2007: 387-sq.). Dado que Polibio no menciona el pugio en su descripción de los pertrechos de los soldados, parece que el puñal no estaba excesivamente extendido antes del siglo I a.C. y que anteriormente era usado principal mente por las tropas auxiliares hispanas (Bishop y Coulston, 2006: 56-57; Poux, 2008: 327-328).

Hasta aquí el breve resumen de los hallazgos de armas en los campamentos romanos alrededor de N umancia. Como ya se ha mencionado, es llamativo el reducido grupo de armas defensivas. Entre ellas aparecen varios discos de coraza, partes de la loriga (lorica hamata) y del cinturón militar, así como un umbo de escudo. En cambio, las armas ofensivas y arrojadizas están bien representadas. J unto a las puntas de lanza, tipológica y cronológicamente bastante irrelevantes, los numerosos hallazgos de pila merecen especial atención. Entre los de lengüeta se encuentran únicamente ejemplares relativamente grandes, que presentan una lengüeta de sujeción plana. En el caso del pilum de enmangue tubular, más robusto, y de las armas arrojadizas estrechamente ligadas a éste, sorprende la cantidad de tipos diferentes con sus variadas posibilidades de utilización. Son abundantes las puntas de pila rotas durante las acciones de combate, que a partir de esta época presentan la forma oblongo-cuadrangular que predominará hasta la época de la R oma imperial. L as puntas de catapul tas desempeñan un papel menor dentro del material hallado, pero presentan una gran variedad de calibres. En cambio, existe una buena representación de otros tipos de puntas de proyectil y de diferentes puntas de flechas, sobre todo con aguijón, pero también con estructura tubular. El número de hojas de espada y de puñal, así como partes de las vainas correspondientes de bronce y hierro, es menor.

Los hallazgos disponibles constituyen la mejor demostración de la presencia de legionarios romanos. En el siglo II a.C., los pertrechos de los legionarios dependía todavía en gran medida de la fortuna personal del soldado, lo que explica la variedad de petos constatados (discos de coraza, loriga) (Cagniart, 2007; 85-sq.; 88-sq.). En este aspecto, la reforma que tuvo lugar en las décadas en torno al año 100 a.C., conocida genéricamente como reforma de M ario, trajo consigo un cambio radical. La mayoría de las espadas y puñales (M iks, 2007: 39), las numerosas puntas de flecha, los proyectiles para hondas, además de las numerosas piezas de cinturones, al gunas de ellos suntuosamente ornamentadas, y las espuelas (L uik, 2002: 91-sq.; Quesada Sanz, 2005: 125-sq.; Bishop y Coulston, 2006: 69-70) prueban que los más variados pueblos participaban como auxiliares en el ejército tanto en infantería como en caballería, portando al parecer armas características de su respectiva procedencia étnica (celtíberos, íberos, celtas). De ello da cuenta, entre otros, un fragmento de la empuñadura de un puñal de frontón encontrada en Renieblas. EI hallazgo de una falcata ibérica en el campamento de Cáceres el Viejo (Ulbert, 1984; tabla 25, 201; véase también Poux, 2008: 329-330) demuestra que los aspectos étnicos desempeñaban un papel importante en el armamento y los pertrechos de las tropas auxiliares aún en el temprano siglo I a.C.

Por lo demás, el lugar de fabricación de casi todos los hallazgos de armas de los campamentos romanos alrededor de Numancia sigue siendo incierto. En cualquier caso, hasta el momento no se ha comprobado con certeza que en dichos campamentos se repararan o incluso se fabricaran armas. El disco de coraza de Castillejo fue confeccionado sin duda alguna en Italia. La inexistencia de partes indiscutibles de gladius resulta bastante sorprendente, más aún tratándose del supuesto lugar de origen de dicha arma. Como demuestra el hallazgo de Šmihel en Eslovenia, el gladius se utilizaba por lo menos desde el temprano siglo II a.C. (Bishop y Coulston, 2006: 54-sq.; K avanagh de Prado y Quesada Sanz, 2007: 70-71; M iks, 2007: 43-sq.; Quesada Sanz, 2007: 391-sq.; Poux, 2008: 316-sq.). También faltan, aparentemente por completo, puntas de proyectiles y de flechas, puntas de arpones, obstáculos para el acercamiento, como los stimuli y tribuli y, lo que llama especialmente la atención, flechas incendiarias es- 
peciales para combatir a los celtíberos, como las que se han hallado, íntegras 0 al menos en fragmentos, en A lesia (Sievers, 2001: 169-sq., tabla 80-83; 85), en el castellum A de La L oma (Peralta Labrador, 2006: 533-534, fig. 4; 2007: 500-501, tabla 3), en Osuna (Quesada Sanz, 2008: 16) 0 en Uxellodunum (Girault, 2007: 268 fig. 8; 275 fig. 14) (panorama general en Poux, 2008: 360-sq.). Es de suponer que, una vez finalizados los combates, se recogería la mayor cantidad de armas posible para volver a emplearlas o bien para al gún otro fin. Sin embargo, estos tipos de armas apenas se usaban o no se usaban en absoluto antes de Numancia. L as numerosas armas arrojadizas encontradas aquí son análogas a las halladas en las fortificaciones de A lesia. En N umancia fal tan, en cambio, las partes típicas de los obstáculos al acercamiento, a pesar de que los sitiadores tendrían que contar también aquí con ataques armados por parte de los sitiados, tal como tan gráficamente lo describe A piano.

L os hallazgos de armas proporcionan sólo un marco general para la datación de los campamentos romanos al rededor de N umancia. Para resolver esta cuestión, es necesario recurrir a otros grupos de hallazgos menores, en primer lugar, las monedas, las fíbulas y la cerámica de vajilla. De este modo resulta una ubicación cronológica de los campamentos romanos al rededor de N umancia que coincide plenamente con el marco temporal que ofrecen las fuentes escritas (L uik, 2002: 16-sq.). Como ya se ha explicado expresamente más arriba, las atribuciones a determinados periodos de los campamentos, tal como propone Schulten, se pueden considerar a menudo problemáticas en cuanto a su metodología. L as dudas acerca de los campamentos IV y $\mathrm{V}$ de Renieblas (según Schulten), cuya ubicación temporal es a la sazón objeto de un controvertido debate, resultan fundadas. Desde un punto de vista puramente estadístico, los hallazgos que se conocen no son cuantitativamente lo bastante ilustrativos como para contar con datos seguros para una datación de los yacimientos. De todos modos, el material conocido actualmente apunta más bien a que estos campamentos se construyeron durante el temprano siglo I a.C., en el contexto histórico del levantamiento de Sertorio, en especial en lo referente al campamento V. Para aclarar de forma definitiva estas y otras cuestiones serán necesarias nuevas excavaciones, en las que, entre otras cosas, deberían descubrirse nuevos hallazgos que provendrán además de contextos estratigráficos seguros, lo que permitirá datarlos con más exactitud. L a esmerada muestra de los descubrimientos de las excavaciones de Schulten debe considerarse aún más relevante en cuanto que sirve de base para futuras investigaciones científicas. A lgunos de los hallazgos de aquella época se pueden calificar de total mente singulares, dado que, a menudo, ha sido muy difícil dar con paralel os o interpretaciones plausibles. Pese a las limitaciones mencionadas, los hallazgos de los campamentos romanos al rededor de Numancia continuarán constituyendo también en el futuro una fuente imprescindible. N unca se val orará lo suficiente la importancia de las agitadas batallas por N umancia, libradas durante años de forma encarnizada, para la evolución del ejército romano (Fernández U riel, 2007: 44sq.; Cagniart, 2007: 81) e incluso para la historia de la República de Roma en general.

\section{BIBLIOGRAFÍA}

Álvarez A rza, R. y M. Cubero A rgente (1998): «L os pila del poblado ibérico de Castellruf». Gladius, XIX: 121-142.

Baatz, D. (1982): «Hellenistische K atapulte aus Ephyra (Epirus)». Athener M itteilungen, XCV II: 211-233.

B aatz, D. (1994): «K atapultbewaffnung und -produktion des römischen H eeres in der frühen und mittleren K aiserzeit», Bauten und K atapulte des römischen H eeres. M AV ORS, R oman A rmy Researches, 11. Stuttgart: 146-168.

Bishop, M . y Coulston, J. C. N . (2006): Roman military Equipment. From the Punic Wars to the F all of Rome. Oxford (second edition). 
Blech, M. (1993): «K rieger aus Estepa», in W. Trillmich et al., Denkmäler der Römerzeit. H ispania Antiqua. Madrid.

Blech, M. (1995): «Schulten und N umantia». M D AI (M ), XXXVI: 38-47.

Blech, M. (2007): «A dolf Schulten, el Instituto A rqueológico A lemán y sus investigaciones en los campamentos romanos», M orillo, A. (ed.), El ejército romano en Hispania. Guía Arqueológica. L eón: 27-38.

Cadiou, F. (2008): Hibera in Terra M iles. Les armées romaines et la conquête de l'Hispanie sous la Republique (218-45 av. J .-C.). B ibliothèque de la Casa de Velázquez, 38. Madrid.

Cagniart, P. (2007): «The Late Republican A rmy (146-30 B.C.)», Erdkamp, P., (ed.), A Companion to the Roman Army. Oxford: 80-95.

Campell, F. (2005): Siege Warfare in the Roman World, 146 BC-AD 378. Oxford.

Cinca, J. L., Velaza, J. y Ramírez Sádaba, J. L. (2003): «U n depósito de proyectiles de catapulta hallado en Calahorra (La Rioja)», AEspA, LXXVI: 263-271.

Connolly, P. (1997): «Pilum, gladius and pugio in the Late Republic». J ournal of Roman M ilitary Equipment Studies, VIII: 41-57.

Connolly, P. (2001-2002): «The pilum from M arius to Nero -a reconsideration of its development and function». J ournal of Roman Military Equipment Studies, XII-X III: 1-8.

Deyber, A. (2008): «Des pointes de traits en fer de «type Numance» (Espagne, province de Soria) à A lésia (Côte-d'Or) et à M ontmartin (Oise)», Poux, M. (éd.) Sur les traces de C ésar. Militaria tardo-républicains en contexte gaulois. Actes de la table ronde du 17 octobre 2002, Glux-En-G lenne - F.58). Glux-en-Glenne. Bibracte, 14: 173-179.

Dobson, M . (2008): The Army of the Roman Republic. The Second Century BC. Polybius and the Camps at Numantia, Spain. Oxford, Oxbow Books.

Dobson, M. y M orales, F. (2008): «M onedas inéditas de los campamentos romanos republicanos de Numancia y Renieblas: consideraciones cronológicas», AE spA, LXXXI: 213-228.

Fernández U riel, P. (2007): «La conquista de la Península I bérica por Roma», M orillo, A. (éd.), El ejército romano en Hispania. Guía Arqueológica. León: 39-54.

Feugère, M. (1993): Les armes de romains de la République à l'Antiquité tardive. Paris.

García Díez, F. y Sáez A bad, R. (2007): «La artillería en la Hispania romana». Sautuola, XIII: 445464.

Girault, J.-P. (2007): «R echerches à la Fontaine de L oulié, Saint-D enis-les-M artel (46). N ouveaux éléments sur la bataille d'U xellodunum», Vaginay, M. et Izac-Imbert, L. (eds.), Les âges du F er dans le Sud-O uest de la F rance. XXVIII e colloque de l'AFEAF (Toulouse, 2004). A quitania, supplément 14/1. B ordeaux: 259-283.

Gómez-Pantoja, J. y F. M orales (2002): «Sertorio en Numancia: una nota sobre los campamentos de Gran A talaya», M orillo, A. (éd.), Arqueología militar romana en Hispania. A nejos de Gladius, 5. Madrid, CSIC: 303-310.

Gruat, Ph. et al. (2003): «D es balles de fronde en plomb de l'armée romaine à Caylus/Puech B oussac (Saint-A ffrique)». Cahiers d'Archéologie Averyronnaise, XVI: 87-96.

Jimeno M artínez, A. (2002): «N umancia: campamentos romanos y cerco de Escipión», AE spA, LXXVI: 159-176.

Jimeno M artínez, A. (2006): «Conquest and Romanization in Celtiberia Ulterior: Numantia as paradigm», Casal A bad, L. et al. (eds.), Early Roman Towns in Hispania Tarraconensis. J ournal of Roman Archaeology, Supplement 62, Portsmouth, R hode Island.

K avanagh De Prado, E. y Quesada Sanz, F. (2007): «L a arqueología militar romana republicana en España: armas, campamentos y campos de batalla; Panorama de la investigación reciente», M orillo, A. (éd.), El ejército romano en Hispania. Guía Arqueológica. L eón: 67-86.

Kuhn-Chen, B. (2007): «A ppianos (A ppian)», Landfester, M ; et Egger, B. (eds.), Geschichte der antiken Texte. Autoren- und Werklexikon. Der N eue Pauly, Supplement 2. Stuttgart, Weimar: 56-58.

Landfester, M. (2007) : «Polybius», Landfester, M; et Egger, B. (eds.), G eschichte der antiken Texte. Autoren- und Werklexikon. Der N eue Pauly, Supplement 2. Stuttgart, Weimar: 494.

Luik, M. (1997): «Die römischen M ilitäranlagen der Iberischen Halbinsel. Von der Republik bis zum A usgang des Prinzipats. Ein Forschungsüberblick», J ahrbuch der Römisch-G ermanischen Zentralmuseums, XLIV: 231-275. 
Luik, M. (2000): «Republikanische Pilumfunde vom «Talamonaccio»«. Archäologisches Korrespondenzblatt, 30: 269-277.

Luik, M. (2002): Die F unde aus den römischen Lagern um Numantia im Römisch-Germanischen Zentralmuseum. K ataloge vor-und frühgeschichtlicher A Itertümer, 31, M ainz, RGZM .

Luik, M. (2007a): Der schwierige Weg zur Weltmacht. Roms E roberung der Iberischen Halbinsel 218219 v. Chr. M ainz, Zweite A uflage.

Luik, M. (2007b): «R enieblas», M orillo, A . (éd.), El ejército romano en Hispania. Guía Arqueológica. León: 286-293.

Luik, M . (2009): «Der feurige K rieg - A rchäologische Forschungen zu den römischen Lagern um Numantia. Ein Ü berblick», M eller, H. (ed.), Schlachtfeldarchäologie. 1. M ittel deutscher Archäologentag 09.- 11.10.2008, Halle (Saale), Tagungen des L andesmuseums für Vorgeschichte $\mathrm{H}$ alle, Band 2, Halle: 49-57.

Luik, M. y M üller, D. (2006): Renieblas, Lager V. Die Ergebnisse der archäologisch-topographischen Vermessungen der J ahre 1997 bis 2001. Iberia A rcheologica, 9, Mainz.

M iks, C. (2007): Studien zur Schwertbewaffnung in der K aiserzeit. Kölner Studien zur A rchäologie der römischen Provinzen, 8. Rahden.

M orales Hernández, F. (1995): La Altiplanicie Soriana. Carta Arqueológica de Soria. Soria.

M oral es Hernández, F. (2007): «Circumvallatio de N umantia», M orillo, A. (éd.), El ejército romano en Hispania. Guía Arqueológica. León: 263-276.

M orillo, Á. (2007): El ejército romano en H ispania. Guía Arqueológica. León.

Peral ta L abrador, E. (2007): «Equipamiento militar romano de la conquista de la antigua Cantabria». Sautuola, XIII: 299-432.

Poux, M. (2008a): «L'empreinte du militaire tardo-républicain dans les faciès mobiliers de La Tène finales», Poux, M. (éd.), Poux, M. (éd.) Sur les traces de César. Militaria tardo-républicains en contexte gaulois. Actes de la table ronde du 17 octobre 2002, Glux-en-Glenne - F.58). Glux-enGlenne. Bibracte, 14: 299-432.

Poux, M. (éd.) Sur les traces de César. Militaria tardo-républicains en contexte gaulois. Actes de la table ronde du 17 octobre 2002, Glux-en-Glenne - F.58). Glux-en-Glenne. Bibracte, 14.

Quesada Sanz, F. (2005): «EI gobierno del caballo montado en la A ntigüedad clásica con espacial referencia al caso de I beria. B ocados, espuelas y la cuestión de la silla de montar, estribos y herraduras», Gladius, XXV: 97-150.

Quesada Sanz, F. (2007): «Hispania y el éjercito romano republicano. Interacción y adopción de tipos metálicos». Sautuola, XIII: 379-402.

Quesada Sanz, F. (2008): «A rmamento romano e ibérico en U rso (Osuna): testimonio de una época». Cuadernos de los Amigos de los M useos de 0 suna, $X: 13-19$.

Schaltenbrand Obrecht, V. (2004): «Die M etallfunde von Filzbach-Vor dem Wald und vom Biberlikopf», Roth-Rubi, K et al. (ed.), Neues zu den «Walenseetürmen». J ahrbuch der Schweizerischen Gesellschaft für U r- und Frühgeschichte, LXXXVII: 33-70.

Schnurbein, S. v. (2002): «A briss der Entwicklung der Römisch-Germanischen Kommission unter den einzel nen Direktoren von 1911 bis 2002», Ber. RGK , LXXXII: 137-289.

Schulten, A . (1927): Die Lager des Scipio. Numantia, III. M unich.

Schulten, A . (1929): Die Lager bei Renieblas. Numantia, IV, M unich.

Schulten, A . (1931): Die Stadt Numantia. N umantia, II, M unich.

Schulten, A . (1953): Cincuenta y cinco años de investigación en E spaña. Reus.

Sáez A bad, R. (2005) : Artillería y poliorcética en el mundo grecorromano, A nejos de Gladius, 8. M adrid, CSIC.

Sievers, S. (2001): «Les armes d'A lésia», Reddé, M. et Schnurbein, S. v. (ed.); A lésia. F ouilles et recherches franco-allemandes sur les travaux militaires romains autour du Mont-Auxois (19911997). M émoires de l'A cadémie des Inscriptions et B elles-L ettres, 22. París: 121-241.

Tomedi, G. (2000): Italische Panzerplatten und Panzerscheiben. Prähistorische Bronzefunde, IV.3. Stuttgart.

Ulbert, G. (1984): Cáceres el Viejo. E in spätrepublikanisches Legionslager in Spanisch-Extremadura. $M$ adrider B eiträge, 11. M ainz.

Velaza, J. et al. (2003): «Nuevo testimonio de las guerras Sertorianas en Calahorra: un depósito de proyectiles de catapulta». Kalakorikos, VIII: 9-30. 
Viand, A . (2008): «L es fragments de cotte de mailles de Vernon. A rmure souple véliocasse ou presence romaine aux portes de l'oppidum?», Poux, M. (éd.) Sur les traces de César. Militaria tardo-républicains en contexte gaulois. Actes de la table ronde du 17 octobre 2002, Glux-en-Glenne - F.58). Glux-en-Glenne. Bibracte, 14: 33-46.

Walbank, F. W. (1979): A historical commentary on Polybius. Vol. 3. Oxford.

Recibido: 22/10/2009

A ceptado: $12 / 04 / 2010$ 